\title{
TRANSIÇÃO METAL-ISOLANTE EM FULLERITOS DOPADOS COM METAIS ALCALINOS
}

\author{
José Carlos Cechin e Ronaldo Mota \\ Departamento de Física - Centro de Ciências Naturais e Exatas \\ UFSM - Santa Maria, RS
}

\section{RESUMO}

Neste artigo estuda-se a ocorrência dos caráters metálico e isolante em fulleritos dopados com metais alcalinos. Em particular, a ocorrência da fase metálica em sistemas dopados com potássio e a não ocorrência desta fase em sistemas dopados com sódio são analisadas via a aproximação de Gutzwiller em um modelo de Hubbard degenerado. Utilizando parâmetros de interação e larguras de bandas derivadas de cálculos INDO-CI na abordagem de aglomerado molecular, em moldes semelhantes aos apresentados anteriormente por Coutinho, Canuto, Mota e Fazzio, as transições metal-isolante são discutidas nesses sistemas. Dos resultados obtidos da relação dos parâmetros de interação e larguras de bandas com a dopagem é possivel entender os comportamentos diversos dos sistemas dopados com potássio e sódio com relação às fases metálicas e isolantes para esses dois sistemas.

\section{ABSTRACT}

In the presel: article of the metallic or insulating characters of alkali doped is studied. In particular, the ocurrence of a metalic phase in potassium doped systems and its nonocurrence in sodium doped systems are analyzed through the Gutzwiller approximation on a degenerate Hubbard model. Using 
interaction and bandwidth parameters derived from INDO-CI calculations in the molecular cluster approach, as presented earlier by Coutinho, Canuto, Mota and Fazzio, the nonmetal-metal transitions are discussed in the systems. From the obtained results for the relation between the derived parameters and doping concentrations, it is possible to help to elucidate the diverse behaviours presented by fullerides doped with potassium and sodium.

\section{INTRODUÇÃO}

A identificação da molécula de $C_{60}$ foi feita em 1985 pelo grupo de Kroto e colaboradores [1], porém, para que estudos experimentais pudessem ser feitos, Krätschmer e colaboradores [2] desenvolveram uma técnica experimental capaz de separar quantidades suficientes de amostras.

Em condições especiais são formados pequenos cristais de fullerenos chamados fulleritos, onde as gaiolas de $C_{60}$ são ordenadas numa estrutura fcc (cúbica de face centrada), a qual pode ser vișualizada como um cubo onde o centro de cada face e os vértices abrigam uma esfera, o que resulta em seis esferas nas faces e oito nos vértices.

Ao doparem-se os fullerenos com metais alcalinos de modo a formar a estequiometria do tipo $A_{3} C_{60}$ a supercondutividade foi observada para $A=K, R b$ e $C s$, enquanto que a fase supercondutora não foi observada para o $\mathrm{Na}_{3} \mathrm{C}_{60}$ [3]. Para as demais estequiometrias esses materiais são semicondutores em temperaturas ambiente.

O surpreendente com os fenômenos associados com os fulleritos dopados com metais alcalinos é o caráter metálico ou isolante desses materiais e a transição metal-isolante observada ao variar-se a concentração do dopante. De acordo com a teoria de banda [4], foi previsto um comportamento metálico para todos estes compostos devido à degenerescência tripla da banda de condução. 
No cristal formado com $C_{60}$ puro o tamanho do gap de uma partícula é de aproximadamente $1,5 \mathrm{eV}$ e a diferença entre os orbitais HOMO ("Highest Occupied Molecular Orbital") de $C_{60}^{+}$(foto emissão) e o orbital LUMO ("Lowest Unoccupied Molecular Orbital") de $C_{60}^{-}$(foto emissão inversa), que está ao redor de $3,5 \mathrm{eV}$, leva a uma energia de correlação $\mathrm{U}$ da ordem de $2 \mathrm{eV}$.

Resultados experimentais e teóricos $[3,5,6]$ confirmam que $\mathrm{U}$ está na faixa de 1 a $3 \mathrm{eV}$. Para a largura de banda estas mesmas referências encontraram $W$ na faixa de 0,2 a $0,6 \mathrm{eV}$. A razão $U / W$ ao redor de quatro sugere que os efeitos de correlação elétron-elétron são muito importantes nesses materiais e que o modelo de elétrons livres deva ser inadequado.

A alta eletroafinidade da molécula $C_{60}$, em torno de $2,5 \mathrm{eV}$ [7], e o baixo potencial de ionização dos metais alcalinos indicariam que para a estequiometria $A_{3} C_{60}$ deveria haver uma completa transferência de carga dos átomos dopantes para a molécula, com os elétrons transferidos ocupando os três sítios LUMO, formando uma banda semipreenchida, resultando num comportamento metálico. Esta hipótese, como veremos, mostrou-se inadequada, mesmo tendo sido corroborada por Martins e Troullier[8] e Novikov e colaboradores [9]. Na verdade, como veremos a seguir, somente uma parte da carga é transferida e com consequências fundamentais no caráter metálico ou isolante desses materiais.

\section{O HAMILTONIANO E A TRANSIÇÃO METAL- NÃO METAL}

Fazemos uso da aproximação adiabática, ou do conhecido método de Born-Oppenheimer [10,11], onde para o movimento dos elétrons só interessa a configuração instantânea dos íons da rede. 
Consideremos então o hamiltoniano

$$
H=\sum_{i}\left(\frac{p_{i}^{2}}{2 m}+V\left(\overrightarrow{r_{i}}\right)\right)-\frac{1}{2} \sum_{i \neq j} \frac{e^{2}}{\left|\overrightarrow{r_{i}}-\overrightarrow{r_{j}}\right|^{2}},
$$

onde $V\left(\overrightarrow{r_{i}}\right)$ é a energia de interação elétron-íon e o último termo representa a interação entre pares de elétrons.

Denominando $h\left(\vec{r}_{i}\right)$ o hamiltoniano de um elétron e de $(1 / 2) \sum_{i j} V_{i j}$ o termo de interação entre os pares de elétrons, escrevemos $H$, em termos em termos dos operadores de segunda quantização, como

$$
H=\sum_{\mu \nu}\langle\mu|h| \nu\rangle a_{\mu}^{\dagger} a_{\nu}+\frac{1}{2} \sum_{\mu \nu \sigma \tau}\langle\mu \nu|v| \sigma \tau\rangle a_{\mu}^{\dagger} a_{\nu}^{\dagger} a_{\tau} a_{\sigma},
$$

onde $\mu, \nu, \sigma$ e $\tau$ representam sítio e spin,

$$
\begin{gathered}
\langle\mu|h| \nu\rangle=\int \Phi_{\mu}^{*}(\vec{r}) h(\vec{r}) \Phi_{\nu}(\vec{r}) d(\vec{r}), \\
\langle\mu \nu|v| \sigma \tau\rangle=\int \Phi_{\mu}{ }^{*}(\vec{r}) \Phi_{\nu}{ }^{*}(\vec{r}) V(\vec{r}-\vec{r}) \Phi_{\sigma}(\vec{r}) \Phi_{\tau}(\vec{r}) d \vec{r} d \vec{r} .
\end{gathered}
$$

onde $\Phi \vec{r}$ são funções orbitais.

Com os autovalores de um elétron dados por $\left\langle\Psi_{\vec{k}}^{\alpha \sigma}|\mathcal{H}| \Psi_{\vec{k}}^{\alpha \sigma}\right\rangle$ iguais a $\epsilon_{\vec{k}}^{\alpha}$, escrevemos

$$
\begin{aligned}
& H=\sum_{\vec{k} \alpha \sigma} \epsilon_{\vec{k}}^{a} a_{\vec{k} \alpha \sigma}^{\dagger} a_{\vec{k} \alpha \sigma}+ \\
& \frac{1}{2} \sum_{\overrightarrow{k_{1}} \overrightarrow{k_{2}}{\overrightarrow{k_{1}}}^{\prime}{\overrightarrow{k_{2}}}^{\prime}} \sum_{\alpha_{1} \alpha_{2} \alpha_{1}^{\prime} \alpha_{2}^{\prime}} \sum_{\sigma \sigma^{\prime}}\left\langle\overrightarrow{k_{1}} \alpha_{1} \overrightarrow{k_{2}} \alpha_{2}\left|\frac{1}{r}\right| \overrightarrow{k_{1}}{ }^{\prime} \alpha_{1}^{\prime} \vec{k}_{2}^{\prime} \alpha_{2}^{\prime}\right\rangle \\
& a_{\overrightarrow{k_{1}} \alpha_{1} \sigma}^{\dagger} a_{\overrightarrow{k_{2} \alpha_{2} \sigma^{\prime}}}^{\dagger} a_{\overrightarrow{k_{1} \alpha_{2} \alpha^{\prime} \sigma^{\prime}}} a_{\overrightarrow{k_{2}{ }^{\prime} \alpha_{1}{ }^{\prime} \sigma^{\prime}}},
\end{aligned}
$$

onde a soma sobre os $\vec{k}$ 's são feitas sobre a primeira zona de Brillouin, e

$$
\left\langle\overrightarrow{k_{1}} \alpha_{1} \overrightarrow{k_{2}} \alpha_{2}\left|\frac{1}{r}\right| \overrightarrow{k_{1}} \alpha_{1}^{\prime} \overrightarrow{k_{2}} \alpha_{2}^{\prime}\right\rangle=e^{2} \int \frac{\left.\Psi_{\overrightarrow{k_{1}}}^{* \alpha_{1}}(\vec{r}) \Psi_{\overrightarrow{k_{2}}}^{* \alpha_{2}}\left(\overrightarrow{r^{\prime}}\right) \Psi_{\overrightarrow{k_{1}}}^{\alpha_{1}^{\prime}}(\vec{r})\right) \Psi_{\overrightarrow{k_{2}}}^{\alpha_{2}^{\prime}}(\vec{r}) d \vec{r} d \vec{r}}{|\vec{r}-\vec{r}|} .
$$

A descrição geral dos elétrons, na representação de Wannier e 
em segunda quantização, pode ser expressa pelo hamiltoniano

$$
H=\sum_{i j} \sum_{\sigma} T_{i j} a_{i \sigma}^{\dagger} a_{j \sigma}+\frac{1}{2} \sum_{i j k l} \sum_{\sigma^{\prime} \sigma}\left\langle i j\left|\frac{1}{r}\right| k l\right\rangle a_{i \sigma}^{\dagger} a_{j \sigma^{\prime}}^{\dagger} a_{l \sigma^{\prime}} a_{k \sigma},
$$

onde $i, j, k$ e $l$ são índices correspondentes aos sítios da rede, $a_{i \sigma}$ e $a_{i \sigma}^{\dagger}$ são operadores de aniquilação e criação para um elétron de spin $\sigma$ no $i$-ésimo sítio da rede. $T_{i j}$ é o elemento de matriz do hamiltoniano (energia cinética e potencial periódico) entre dois orbitais em sítios diferentes, dado por

$$
T_{i j}=\left\langle\Phi\left(\vec{r}-\vec{R}_{i}\right)\left(\frac{p^{2}}{2 m}\right) \Phi\left(\vec{r}-\vec{R}_{j}\right)\right\rangle,
$$

onde $\Phi\left(\vec{r}-\vec{R}_{i}\right)$ e $\Phi\left(\vec{r}-\vec{R}_{j}\right)$ são funções de Wannier dos sítios $i$ e $j$, respectivamente.

No hamiltoniano (7) ao considerarmos as integrais de quatro centros $\left\langle i j\left|\frac{1}{r}\right| k l\right\rangle$, por serem estas calculadas em termos de funções de Wannier bastante localizadas, retemos em primeira aproximação, o termo dominante $\left\langle i i\left|\frac{1}{r}\right| i i\right\rangle=U$. Nestas condições

$$
H=\sum_{i j \sigma} T_{i j} a_{i \sigma}^{\dagger} a_{j \sigma}+\frac{1}{2} U \sum_{i \sigma} a_{i \sigma}^{\dagger} a_{i \sigma} a_{i-\sigma}^{\dagger} a_{i-\sigma} .
$$

O hamiltoniano acima, também conhecido como hamiltoniano Hubbard [11, 12], contém o mínimo de propriedades necessárias para descrever tanto um comportamento localizado quanto de banda, especialmente o comportamento intermediário entre esses dois limites.

\section{APROXIMAÇÃO DE GUTZWILLER}

Gutzwiller [13] apresenta um estudo acerca da função de onda do estado de mais baixa energia para elétrons em uma banda s estreita, a qual é investigada para uma densidade arbitrária de elétrons e para uma interação de qualquer intensidade. A característica principal da aproximação proposta por ele reside em reduzir os complicados cálculos a uma contagem razoavelmente simples dos tipos de configurações acessíveis ao sis- 
tema atribuindo os pesos apropriados a cada uma delas. Assim, o cálculo de qualquer valor esperado depende, de acordo com Gutzwiller, diretamente do número de configurações participantes e de seus pesos relativos.

Dos $L$ sítios da rede um conjunto $G=\left(g_{1}, \ldots, g_{m}\right)$ representam o conjunto de $m$ sítios da rede ocupados por partículas com spin para cima. Em contrapartida $\Gamma=\left(\gamma_{1}, \ldots, \gamma_{\mu}\right)$ representa um conjunto de $\mu$ sítios da rede ocupados por partículas com spin para baixo.

Uma configuração $\Phi_{G \Gamma}$ correspondente a estes dois conjuntos é formada da seguinte maneira

$$
\Phi_{G, \Gamma}=\prod_{G} a_{g}^{\dagger} \prod_{\Gamma} a_{\gamma}^{\dagger} \Phi_{0}
$$

onde $\Phi_{0}$ é o estado de vácuo. A seguir formamos o estado correlacionado em termos das amplitudes $A_{G \Gamma}$ pela expansão

$$
\Psi=\sum_{G \Gamma} A_{G \Gamma} \Phi_{G \Gamma}
$$

onde o somatório abrange todos conjuntos distintos $G$ e $\Gamma$. As amplitudes $A_{G \Gamma}$ podem ser escolhidas arbitrariamente como números reais ou mesmo complexos.

Afim de calcularmos os valores esperados da energia para $\Psi$, precisamos somente das funções densidade de primeira e segunda ordens, $\rho_{1}$ e $\rho_{2}$. Porém, às vezes, é interessante examinar a função densidade nésima em termos de seus argumentos, os quais agora escolhemos serem os sítios da rede e direções de spin, ou seja

$$
\rho_{n}\left(h_{1 \uparrow}, \ldots, h_{n \uparrow} ; f_{1 \uparrow}, \ldots, f_{n \uparrow}\right)=(n !)^{-1}\left(\Psi\left|a_{h_{1 \uparrow}}^{\dagger}, \ldots, a_{h_{n \uparrow}}^{\dagger} a_{f_{1 \uparrow}}, \ldots, a_{f_{n \uparrow}}\right| \Psi\right)
$$

$\mathrm{O}$ conjunto de fases a ser adotado vem de alguma função de onda não correlacionada que é o produto antissimetrizado de funções de Bloch

$$
\Phi=\prod_{k} a_{k \uparrow}^{\dagger} \prod_{K} a_{K \downarrow}^{\dagger} \Phi_{0} .
$$


O conjunto $(k)$ é suposto ser o volume contido dentro de alguma superfície de Fermi S e similarmente o conjunto $(K)$ é relativo ao interior de alguma superfície de Fermi $\sum$ no espaço recíproco. Os coeficientes na expansão de $\Psi$ na Eq.(11) são dados pelo produto de dois determinantes

$$
\left(\begin{array}{c|ccc}
\mathcal{L}^{-1 / 2} e^{i k g} & k_{1} & \cdots & k_{m} \\
g_{1} & \cdots & g_{m}
\end{array}\right)\left(\begin{array}{l|lll}
\mathcal{L}^{-1 / 2} e^{i k \gamma} & K_{1} & \cdots & K_{\mu} \\
\gamma_{1} & \cdots & \gamma_{m}
\end{array}\right),
$$

onde usou-se a representação

$$
\left(f(x, y)\left|\begin{array}{ccc}
x_{1} & \cdots & x_{n} \\
y_{1} & \cdots & y_{m}
\end{array}\right|=\left|\begin{array}{ccc}
f\left(x_{1}, y_{1}\right) & \cdots & f\left(x_{1}, y_{m}\right) \\
f\left(x_{2}, y_{1}\right) & \cdots & f\left(x_{2}, y_{m}\right) \\
\vdots & & \vdots \\
f\left(x_{n}, y_{1}\right) & \cdots & f\left(x_{n}, y_{m}\right)
\end{array}\right| .\right.
$$

Se os conjuntos $(k)$ e $(K)$ forem escolhidos de tal maneira que incluam sempre $k$ e $-k$ simultaneamente, e similarmente $K \mathrm{e}-K$, cada um dos dois determinantes é um número real vezes alguma potência fixa de $i$.

As amplitudes $A_{G \Gamma}$ da funçăo de onda correlacionada $\Psi$ são agora dadas por

$A_{G \Gamma}=B_{G \Gamma} \eta^{\nu}\left(L^{-\frac{1}{2}} \exp (i k g) \mid \begin{array}{ccc}k_{1} & \cdots & k_{m} \\ g_{1} & \cdots & g_{m}\end{array}\right)\left(L^{-\frac{1}{2}} \exp (i K \gamma) \mid \begin{array}{ccc}K_{1} & \cdots & K_{\nu} \\ \gamma_{1} & \cdots & \gamma_{\nu}\end{array}\right)$

onde $\nu$ é o número de sítios da rede idêndicos entre os conjuntos $\left(g_{1}, \ldots, g_{m}\right)$ e $\left(\gamma_{1}, \ldots, \gamma_{\mu}\right), B_{G \Gamma}$ é um número real e positivo e $\eta$ dá o peso de cada configuração. Então

$$
\left|A_{G \Gamma}\right|^{2}=B_{G \Gamma}^{2} \eta^{2 \nu}\left(w\left(g^{\prime}-g^{\prime \prime}\right) \mid \begin{array}{ccc}
g_{1} & \cdots & g_{m} \\
g_{1} & \cdots & g_{m}
\end{array}\right)\left(W\left(\gamma^{\prime}-\gamma^{\prime \prime}\right) \mid \begin{array}{ccc}
\gamma_{1} & \cdots & \gamma_{\nu} \\
\gamma_{1} & \cdots & \gamma_{\nu}
\end{array}\right),
$$

onde $w\left(g^{\prime}-g^{\prime \prime}\right)$ e $W\left(\gamma^{\prime}-\gamma^{\prime \prime}\right)$ são as funçöes propagações 


$$
\begin{aligned}
w(h-f) & =\frac{1}{L} \sum_{k} \exp (i k h-i k f), \\
W(h-f) & =\frac{1}{L} \sum_{K} \exp (i K h-i K f) .
\end{aligned}
$$

Afora contribuirem com um sinal a $A_{G \Gamma}$, os determinantes que aparecem no lado direito de $A_{G \Gamma}$ contribuem para o valor absoluto da amplitude dos determinantes que aparecem do lado direito de $\left|A_{G \Gamma}\right|^{2}$. Os últimos devem incluir o efeito do assim chamado buraco de "exchange".

Este efeito, tem portanto, uma influência perniciosa na energia cinética em termos do potencial do cristal os quais são determinados por $\rho_{1}\left(h_{\uparrow}, f_{\uparrow}\right)$. Sendo mais explícitos, ao redor de um sítio $\uparrow$ haverá uma redução de sítios $\downarrow$ e uma tendência de encontrarem-se dominantemente sítios $\downarrow$. Para evidenciar isto, compare a expressão para $\eta=1$ e $B_{G \Gamma}=1$ (sem correlação),

$$
\begin{aligned}
\rho_{1}\left(h_{\uparrow}, f_{\uparrow}\right)= & \sum_{g_{2} \cdots g_{m}}\left(w\left(g^{\prime}-g^{\prime \prime}\right) \mid \begin{array}{ccc}
h g_{2} & \cdots & g_{m} \\
f g_{2} & \cdots & g_{m}
\end{array}\right) \\
& \sum_{\Gamma}\left(W\left(\gamma^{\prime}-\gamma^{\prime \prime}\right) \mid \begin{array}{ccc}
\gamma_{1} & \cdots & \gamma_{\mu} \\
\gamma_{1} & \cdots & \gamma_{\mu}
\end{array}\right)^{x}= \\
= & w(h-f),
\end{aligned}
$$

com a expressão para $\eta=0$ (fortíssima correlação),

$$
\begin{gathered}
\rho_{1}\left(h_{\uparrow}, f_{\uparrow}\right)=\sum_{g_{2} \cdots g_{m}}\left(w\left(g^{\prime}-g^{\prime \prime}\right) \mid \begin{array}{ccc}
h g_{2} & \cdots & g_{m} \\
f g_{2} & \cdots & g_{m}
\end{array}\right) \times \\
\sum_{\Gamma \neq(f G h)} B_{f G \Gamma} B_{h G \Gamma}\left(W\left(\gamma^{\prime}-\gamma^{\prime \prime}\right) \mid \begin{array}{ccc}
\gamma_{1} & \cdots & \gamma_{\mu} \\
\gamma_{1} & \cdots & \gamma_{\mu}
\end{array}\right),
\end{gathered}
$$

onde o somatório sobre $\Gamma$ inclui somente tais conjuntos de sítios da rede $\gamma_{1}, \ldots, \gamma_{\mu}$ os quais não coincidem com quaisquer dos sítios $f_{1}, g_{2}, \ldots, g_{m}, h$.

Primeiramente, observe que a soma sobre $\Gamma$ tem $\left(\begin{array}{c}L-m-1 \\ \mu\end{array}\right)$ 
termos quando $h \neq f \mathrm{e}\left(\begin{array}{c}L-m \\ \mu\end{array}\right)$ quando $h=f$. O valor de $\rho_{1}\left(h_{\uparrow}, f_{\uparrow}\right)$ é fixado pela normalização em $m / L=w(f-f)$. O valor de $\rho_{1}\left(h_{\uparrow}, f_{\uparrow}\right)$ usualmente será menor do que $w(h-f)$, se $h \neq f$, porque a soma sobre $\Gamma$ tem menos termos, na verdade por um fator de $\left(\begin{array}{c}L-m-\mu \\ \mu\end{array}\right)$. A correlação afeta, portanto, a transferência de elétrons entre sítios da rede mais do que sua chance de estarem em qualquer sítio particular da mesma.

É então concebível que $\rho_{1}\left(h_{\uparrow}, f_{\uparrow}\right)$ se torne bastante independente da escolha particular dos vetores de onda $K$ que aparecem em $W$, isto é, independente da região do espaço recíproco ocupado por elétrons com spin para baixo e um valor típico de $\rho_{1}\left(h_{\uparrow}, f_{\uparrow}\right)$ é obtido através de

$$
\begin{gathered}
\sum_{\Gamma \neq G}\left|B_{G \Gamma}\right|^{2}\left(W\left(\gamma^{\prime}-\gamma^{\prime \prime}\right) \mid \begin{array}{ccc}
\gamma_{1} & \cdots & \gamma_{\mu} \\
\gamma_{1} & \cdots & \gamma_{\mu}
\end{array}\right)=1, \\
\sum_{\Gamma \neq(f G h)} B_{f G \Gamma} B_{h G \Gamma}\left(W\left(\gamma^{\prime}-\gamma^{\prime \prime} \mid \begin{array}{ccc}
\gamma_{1} & \cdots & \gamma_{\mu} \\
\gamma_{1} & \cdots & \gamma_{\mu}
\end{array}\right)=\frac{L-m-\mu}{L-m} .\right.
\end{gathered}
$$

As duas últimas fórmulas expressam a idéia de que os coeficientes podem ser escolhidos tal que possam dar valores simples para as funções densidade $\rho_{n}$, a escolha sendo limitada pelo número total de configurações que contribuem para uma dada $\rho_{n}$. Acredita-se que as propriedades de $\rho_{n}$ obtidas desta maneira sejam típicas da função de onda correlacionada de interesse no nosso problema. A presença dos elétrons com spin para baixo meramente restringem a liberdade de movimento dos elétrons com spin para cima, sem no entanto destruir as relações de fase dos últimos.

De acordo com Gutzwiller [13], a constante que aparece no cálculo da densidade é dada em função do número de configurações vêzes o peso destas e ainda vezes o maior termo da série hipergeométrica dado por 


$$
\begin{gathered}
F\left(\alpha-m, \beta-\mu ; L-m-\mu+\delta ; \eta^{2}\right)= \\
\frac{\Gamma(m-\alpha+1) \Gamma(\mu-\beta-1) \Gamma(L-m-\mu+\delta+\nu) \nu !}{\Gamma(m-\alpha-\nu+1) \Gamma(\mu-\beta-\nu+1) \Gamma(L-m-\mu+\delta+\nu) \nu !},
\end{gathered}
$$

desde que $\alpha, \beta$ e $\delta$ sejam da ordem de 1 . Lembramos que a função hipergeométrica tem como propriedade ser igual a 1 quando um de seus argumentos é zero.

Sendo $\nu$ o número de sítios duplamente ocupados, podemos usar a condição empírica determinada por Guggenheim [14].

$$
\left[\frac{(m-\nu)(\mu-\nu)}{\nu(L-m-\mu+\nu)}\right] \eta^{2}=1 .
$$

onde a quantidade $\eta^{2}$ desempenha portanto, a mesma funçãc que o fator de Boltzmann na lei da ação das massas. Deveras, $m-\nu$ e $\mu-\nu$ são os números médios de partículas dissociadas com spin para cima $\mathrm{e}$ spin para baixo respectivamente, enquanto $\nu$ é o número médio de pares de spins ligados e $L-m-\mu+\delta$ é o número médio de sítios vazios da rede. $\mathrm{O}$ método aqui apresentado pode ser comparado com o método quasiquímico da teoria das misturas [14]. Com $\eta$ variando de 0 a 1 ou seja, entre estados fortemente e não correlacionados, $\nu$ varia de 0 (forte correlação) a $m \mu / L, o$ qual é o número de sítios duplamente ocupados quando não há correlação Este pode ser assumido como uma resultado empírico.

Vejamos para $\mu=m, h_{1}=f_{1}, \ldots, h_{m}=f_{m}$. Se existirem $\nu$ sítios duplamente ocupados, existe um total de $m !(L-m) ! / \nu !(m-\nu) !(\mu-\nu) !(L-$ $m-\mu+\nu)$ ! configurações contribuindo para $\rho_{n}$. A constante que aparece na Eq. da densidade é dada por

$$
\begin{gathered}
\sum_{\nu=0} \frac{\eta^{2 \nu} m !(L-m) !}{\nu !(m-\nu) !(\mu-\nu) !(L-m-\mu+\nu) !}= \\
=\frac{(L-m) !}{\mu !(L-m-\mu) !} F\left(-m,-\mu ; L-m-\mu+1 ; \eta^{2}\right) .
\end{gathered}
$$

Sendo que todas quantidades a serem calculadas daqui para diante, devem ser divididas por essa normalização. 
Examinemos agora a situação de nosso interesse onde $\mu=m$, $h_{1} \neq f_{1}, h_{2}=f_{2}, \ldots, h_{m}=f_{m}$, isto é, o caso de vizinhos mais próximos, considerando a operação $a_{f \uparrow}^{\dagger} a_{g \uparrow}$. As possibilidades distintas que levam a diferentes contribuições da constante são: nenhum dos sítios pode estar duplamente ocupado, um mas não o outro pode estar duplamente ocupado e finalmente ambos podem estar duplamente ocupados.

Caso a): Este caso não envolve spin pára baixo. Não há formação de pares $\nu=0$ e $\eta^{2 \nu}=1$. Um spin para cima foi envolvido na operação. Logo o número de configurações é dado por

$$
\begin{gathered}
\frac{(m-1) !(L-m-1) !}{(m-1) !(\mu) !(L-m-\mu-1) !}= \\
\frac{(L-m-1) !}{\mu !(L-m-\mu-1) !(L-m)(L-m-\mu)} \frac{(L-m)(L-m-\mu)}{(L-m)}= \\
=\frac{(L-m) !}{\mu !(L-m-\mu) !} \frac{(L-m-\mu)}{(L-m)}
\end{gathered}
$$

e $F=F\left(1-m,-\mu ; L-m-\mu ; \eta^{2}\right)$, portanto,

$$
C a=\frac{(L-m) !}{\mu !(L-m-\mu) !} \frac{(L-m-\mu)}{(L-m)} F\left(1-m,-\mu ; L-m-\mu ; \eta^{2}\right) .
$$

Caso b): Apresenta dois subcasos, ainda que equivalentes no resultado final, sendo que nesta instância um par é envolvido, assim como um spin para cima e um spin para baixo. O peso passa a ser $2 \eta$. O número de configurações é dado por

$$
\frac{(m-1) !(L-m-1) !}{(m-1) !(\mu-1) !(L-m-\mu) !} \frac{\mu(L-m)}{\mu(L-m)}=\frac{(L-m) !}{\mu !(L-m-\mu)} !\left(\frac{\mu}{L-m}\right),
$$

e $F=F\left(1-m, 1-\mu ; L-m-\mu+1 ; \eta^{2}\right)$, portanto,

$$
C b=\frac{(L-m) !}{\mu !(L-m-\mu) !} \frac{(\mu)}{(L-m)} 2 \eta F\left(1-m, 1-\mu ; L-m-\mu+1 ; \eta^{2}\right) .
$$

Caso c): É uma transição, "hopping", entre dois sítios ambos ocupados com spin para baixo. O peso passa a ser $\eta^{2}$.

O número de configurações será dado por:

$$
\frac{(m-1) !(L-m-1) !}{(m-1) !(\mu-2) !(L-m-\mu+1) !} \frac{\mu(\mu-1)}{(L-m) \mu(\mu-1)(L-m)}=
$$




$$
=\frac{(L-m) !}{\mu !(L-m-\mu) !} \frac{\mu(\mu-1)}{(L-m)(L-m-\mu+1)},
$$

e $F=F\left(1-m, 2-\mu ; L-m-\mu+2 ; \eta^{2}\right)$, portanto

$$
\begin{gathered}
C_{c}=\frac{(L-m) !}{\mu !(L-m-\mu) !(L-m)(L-m-\mu+1)} \frac{\mu(\mu-1)}{(L-m} \eta^{2} \times \\
F\left(1-m, 2-\mu ; L-m-\mu+2 ; \eta^{2}\right) .
\end{gathered}
$$

A constante $\mathrm{C}$ é então a soma das três expressões acima obtidas divididas pela normalização. Obtemos então para $\rho_{1}$, a qual pode ser expresso por

$$
\rho_{1}\left(h_{\uparrow}, f_{\uparrow}\right)=q w(h-f),
$$

onde $q$ é o fator de correlação, ou seja, $q=1$ para o sistema não correlacionado, resultando em geral

$$
q=\frac{(m-\nu)(L-m-\mu+\nu)}{m(L-m)}\left[1+\frac{(\mu-\nu) \eta}{L-m-\mu+\nu}\right]^{2} .
$$

De forma análoga ao desenvolvimento anterior, obtemos

$$
\begin{aligned}
\rho_{2}\left(g_{\uparrow}, g_{\downarrow} ; g_{\uparrow}, g_{\downarrow}\right)= & \frac{m \mu}{L(L-m-\mu+1)} \eta^{2} \times \\
& \frac{F\left(1-m, 1-\mu ; L-m-\mu+2 ; \eta^{2}\right)}{F\left(-m,-\mu ; L-m-\mu+1 ; \eta^{2}\right)} \\
= & \frac{m \mu}{L(L-m-\mu+1)} \eta^{2} \times \\
& \frac{(m-\nu)(\mu-\nu)(L-m-\mu+1)}{m(\mu-2)(L-m-\mu+\nu+1)} .
\end{aligned}
$$

Sendo $\eta^{2}=\nu(L-m-\mu+\nu) /(m-\nu)(\mu-\nu)$ então

$$
\begin{gathered}
\rho_{2}=\frac{1}{L(L-m-\mu+1)} \times \\
\frac{\nu(L-m-\mu+\nu)}{(m-\nu)(\mu-\nu)} \frac{(m-\nu)(\mu-\nu)(L-m-\mu+1)}{(L-m-\mu+\nu+1)}=\frac{\nu}{L} .
\end{gathered}
$$

Para o hamiltoniano

$$
H=\sum T_{i j} a_{i \sigma}^{\dagger} a_{j \sigma}+U \sum a_{i \sigma}^{\dagger} a_{i \sigma} a_{i-\sigma}^{\dagger} a_{i-\sigma},
$$

calcularemos o valor esperado da energia 


$$
\langle E\rangle=\frac{\langle\Psi|H| \Psi\rangle}{\langle\Psi \mid \Psi\rangle},
$$

ou seja,

$$
\langle E\rangle=\sum T_{i j} \frac{\left\langle\Psi\left|a_{i \sigma}^{\dagger} a_{j \sigma}\right| \Psi\right\rangle}{\langle\Psi \mid \Psi\rangle}+U \sum \frac{\left\langle\Psi\left|a_{i \sigma}^{\dagger} a_{i \sigma} a_{i-\sigma}^{\dagger} a_{i-\sigma}\right| \Psi\right\rangle}{\langle\Psi \mid \Psi\rangle}
$$

Assumindo $T_{i j}=\mathcal{E}$, a energia média por elétron sem correlação, e utilizando a Eq.(33) e a Eq.(36), o valor esperado médio da energia por sítio $\langle\bar{H}\rangle$ resulta como

$$
\langle\bar{H}\rangle=x q \mathcal{E}+\bar{\nu} U
$$

onde $\bar{\nu}=\nu / L$ e $\bar{x}$ é o número de elétrons.

Se eliminarmos o fator peso $\eta$, substituindo a Eq.(25) na Eq.(34), para o caso $m=\mu$, obtém-se

$$
q=\frac{(m-\nu)\left[(L-2 m+\nu)^{1 / 2}+(\nu)^{1 / 2}\right]^{2}}{m(L-m)} .
$$

Paro o caso em que $\bar{m}=\bar{\mu}=\frac{1}{2}$ (nosso sistema é não magnético), $\operatorname{com} \bar{\mu}=\frac{\mu}{L}$ e $\bar{m}=\frac{m}{L}$, resulta em $q=16 \bar{\nu}\left(\frac{1}{2}-\bar{\nu}\right)$. Temos portanto para o valor esperado da energia a expressão

$$
\langle\bar{E}\rangle=16 x \mathcal{E}\left(\frac{1}{2} \bar{\nu}-\bar{\nu}^{2}\right)+\bar{\nu} U .
$$

Para encontrar o número de sítios da rede duplamente ocupados que minimizam $\langle\bar{E}\rangle$, fazemos

$$
\frac{\partial\langle\bar{E}\rangle}{\partial \bar{\nu}}=0=16 x \mathcal{E}\left(\frac{1}{2}-2 \bar{\nu}\right)+U,
$$

donde extraímos

$$
\bar{\nu}=\frac{1}{4}\left[1+\frac{U}{8 x \mathcal{E}}\right] .
$$

Sabemos que $0 \leq x \geq 2 N$, onde $N$ é a degenerescência $(N=3$ no nosso caso) e $\mathcal{E}=[(x-2 N) / 4 N] W$, sendo $W$ a largura de banda. $\mathcal{E}$ pode ser menor do que zero, logo podemos escrever $|\mathcal{E}|=[(2 N-x) / 4 N] W$, portanto 


$$
\bar{\nu}=\frac{1}{4}\left[1-\frac{U}{8 x|\mathcal{E}|}\right] .
$$

Para um valor médio de pares nulo, o que implica na ausência de "hopping" e portante a fase é isolante, encontramos $U=8 x[(2 N-$ $x) / 4 N] W$. A razão $U / W=2 x(2 N-x) / N$ é o que buscamos comparar com cálculos de estrutura eletrônica. Para uma degenerescência tripla ( $\mathrm{N}=3$ ) e um número de elétrons $x=3$ (semi-preenchimento), obtemos $U / W=6$.

Para os valores de $\bar{m}=\bar{\nu} \neq \frac{1}{2}$, resulta

$$
\frac{\partial\langle\bar{E}\rangle}{\partial \bar{\nu}}=x \mathcal{E} \frac{\partial q}{\partial \bar{\nu}}+U
$$

onde

$$
\frac{\partial q}{\partial \bar{\nu}}=-\frac{\left(\sqrt{1-2 \bar{m}+\bar{\nu}}+\sqrt{\bar{\nu}}^{2}(-\sqrt{1-2 \bar{m}+\bar{\nu}} \sqrt{\bar{\nu}}+\bar{m}-\bar{\nu})\right.}{\bar{m}(-1+\bar{m}) \sqrt{1-2 \bar{m}+\bar{\nu}} \sqrt{\bar{\nu}}}
$$

Para encontrar o número de sítios da rede duplamente ocupados que minimizam $\langle\bar{E}\rangle$ igualamos a Equação (46) a zero

$$
x \mathcal{E} \frac{\partial q}{\partial \bar{\nu}}+U=0
$$

onde $\partial q / \partial \bar{\nu}$ é expresso pela Equação (47).

Assumimos anteriormente que a transição metal-isolante no caso de semi-preenchimento equivale ao caso de número de pares nulo, o que é absolutamente correto. Tal suposição deve ser válida aproximadamente também para estados próximos do semi-preenchimento, ou seja, a existência de saltos está seguramente associada à ocorrência de estados duplamente ocupados, salvo para dopagens muito pequenas onde a existência de estados vazios abundantes permitiria "hopping" sem dupla ocupação.

Por fim, assumindo como válida em geral a relação entre transição metal-isolante e a não ocorrência de pares, podemos escrever a relação entre $U$ crítica $\left(U_{c}\right)$ e a largura de banda $(W)$ como: 


$$
\frac{U}{W}=\frac{x[2 N-x]}{4 N} \frac{\partial q}{\partial \bar{\nu}}
$$

Para o caso particular onde $\bar{m}=\frac{1}{2}, \frac{\partial q}{\partial \bar{\nu}}=8$ o resultado anterior de $\frac{U}{W}=6$ é recuperado, como esperado

\section{ESCOLHA DO AGLOMERADO}

A utilização de aglomerados moleculares tem sido uma abordagem muito útil no estudo da estrutura eletrônica de sólidos [15]. Mais recentemente, Coutinho e colaboradores [16] apresentaram cálculos de estrutura eletrônica dos materiais que estamos analisando no presente trabalho utilizando aglomerados moleculares para simular o comportamento dos mesmos.

A grande eletroafinidade do $C_{60}$ e o baixo potencial de ionização dos alcalinos pode, à primeira vista, sugerir uma completa transferência de carga dos átomos alcalinos para o fullereno. No entanto, nossos resultados mostram que a nova banda é devida basicamente ao $C_{60}$, através do orbital $t_{1 u}$, o qual descende de energia. Somente aproximadamente $10 \%$ da banda é devido ao $\mathrm{K}$ e $25 \%$ ao $N_{a}$ nas dopagens $K_{3} C_{60}$ e $\mathrm{Na}_{3} C_{60}$ respectivamente, sendo a maior parte devido ao rebaixamento de orbitais do $C_{60}$. A maior contribuição do $N a$, comparada com o $K$, evidencia que o $K$ é mais delocalizado do que o $N a$, implicando portanto em maior possibilidade de transferência eletrônica.

Uma abordagem qualitativa envolvendo o parâmetro de rede $a$ também aponta na mesma direção. Isto pode ser expresso em experimentos comparativos da correlação simples entre $a$ e a temperatura de transição observada $\left(T_{C}\right)$ para fulleritos com diferentes misturas de $N a, K, R_{b}$ e $C_{S}$. Chen et al [17] obtiveram como resultado que para diferentes materiais 
$A_{3} C_{60}$, todos com a mesma estrutura, a supercondutividade não é observada para o $\mathrm{Na}_{3} \mathrm{C}_{60}$ e para cátions maiores o parâmetro de rede cresce com $T_{C}$ numa taxa de $55 K / \AA$. Se associarmos que, em geral, para parâmetros de rede maiores teremos transferências eletrônicas crescentes para sistemas dopados com alcalinos, resulta que o $N a$ quase não transfere as cargas e c $K, R b$ e $C s$ transferem crescentemente nesta ordem.

Cálculos numéricos de densidades de estados para o aglomerado $\left(C_{60}\right)_{3} N a_{8}$, similares aos apresentados anteriormente por Coutinho et al [16], permitem que façamos comparação para os resultados de $U c$, a energia de correlação crítica, e $W$, a largura de banda associada, para ambos os sistemas $(\mathrm{Na}$ e $\mathrm{K})$ e discutir os estados metálico ou isolante obtidos para cada um dêles.

Assumindo o valor efetivo de $U c$ como associado à diferença entre as energias orbitais HOMO e LUMO, obtemos para $K, U=2,69 \mathrm{eV}$ e $W=0,50 \mathrm{eV}$, e para o $N a, U=2,72 \mathrm{eV}$ e $W=0,32 \mathrm{eV}$, resultando em $U / W$ igual a 5,6 e 8,50 para o $K$ e o $N a$ respectivamente.

Na Fig.(1) apresentamos os números obtidos para a razão $U / W$ para o $x$ apropriado no diagrama de fase para a transição Mott-Hubbard metal-isolante. A linha de transição é traçada usando as Equações (47) e (49) para $N=3$. É possível portanto concluir, a partir da Fig.(1), que para $x \cong 3$, os sistemas dopados com sódio são isolantes e, por sua vez, os dopados com potássio são metálicos.

\section{DISCUSSÃO E CONCLUSÕES}

Utilizando o resultado obtido por J. P. Lu [18], apresentamos em artigo publicado recentemente [19] uma discussão das transições metalisolante observadas em fulleritos dopados com metais alcalinos, via a uti- 


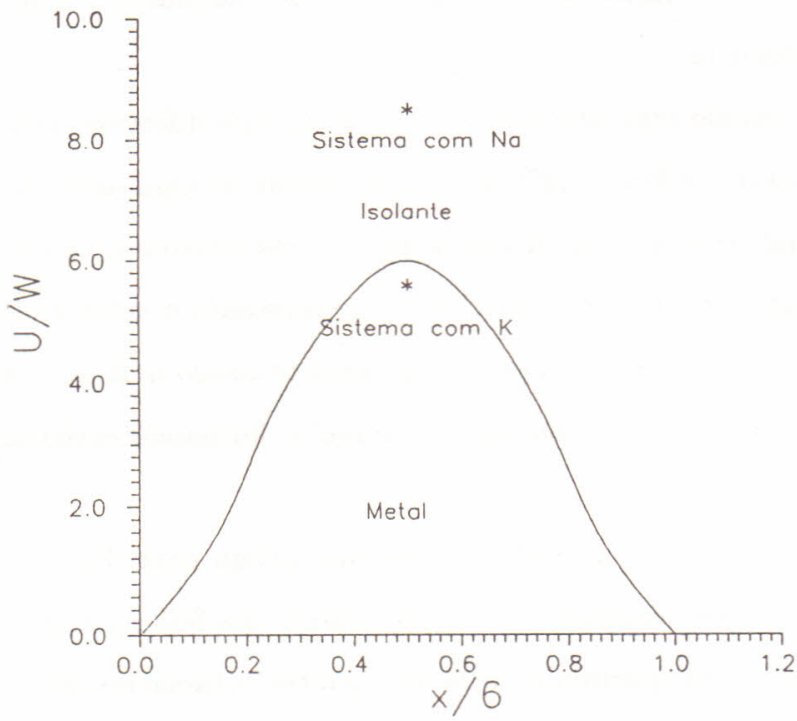

Figura 1: Diagrama de fase para a transição Mott-Hubbard metal-isolante e respectivas posições dos sistemas dopados com potássio e sódio.

lização de parâmetros derivados de cálculos INDO-CI na abordagem de aglomerado molecular.

No presente artigo, utilizando a mesma aproximação de Gutzwiller, uma dedução própria é apresentada, gerando um resultado final para a energia de correlação crítica diferente daquela obtida por J. P. Lu [18]. Utilizando parâmetros obtidos por Coutinho, Canuto, Mota e Fazzio [16], apresentamos aqui uma possível explicação para a ocorrência do caráter metálico em sistemas dopados com potássio e a não ocorrência para materiais dopados com sódio.

No presente artigo a questão principal que nos dispusemos a responder refere-se ao fato que todos os cristais estáveis com estequiometria $A_{3} C_{60}(A=N a, R b, K, C s)$ apresentam a mesma estrutura cristalina e do 
ponto de vista eletrônico todos os alcalinos são semelhantes, com um único elétron de valência.

Usando uma abordagem completamente diferente da aqui apresentada, Garavelli e Ferraz [20] fizeram um estudo comparativo do parâmetro $r_{s}$, o qual representa a distância média intereletrônica nos fullerídeos dopados, com o valor de $r_{s}$ crítico, o qual representa o valor limite para o qual um cátion formado de um metal alcalino colocado num gás de elétrons irá capturar um elétron da banda de condução, formando então um estado ligado.

Concluindo, portanto, o presente artigo contribui com um estudo explicativo dos caráters metálico e isolante dos fulleritos dopados com metais alcalinos. Em particular para os dopantes potássio ou sódio, a aproximação de Gutzwiller mostrou-se adequada. Utilizando-se parâmetros de cálculos de estrutura eletrônica INDO-CI, na abordagem de aglomerado molecular, através de um modelo de Hubbard degenerado, foi possível elucidar os comportamentos diversos dos sistemas dopados com esses dois elementos com relação às fases metálica e isolante. A posição de cada um deles, do fullerito dopado com $N_{a}$ (isolante) e dopado com $K$ (metálico), para meia ocupação, como visto no diagrama de fase da Fig.(1), evidencia exatamente a consistência da abordagem proposta e sua concordância com os resultados experimentais conhecidos.

\section{Referências}

[1] H. W. Kroto, J. R. Heath, S. C. O'Brien, R. F. Curl e E. Smalley, Nature 318, 162 (1985).

[2] W. Krätschmer, L. O. Lamb, K. Fostiroupolos e R. Huffuman, Nature 347, 354 (1990). 
[3] J. H. Weaver, J. Phys. Chem. Solid 53, 1433 (1992).

[4] S. Saito e A. Oshiyana, Phys. Rev. Lett. 66, 2637 (1991); S. C. Erwind e W. E. Pickett, Science 254, 842 (1992); W. E. Pickett, Solid State Phys. 48, 225 (1994).

[5] C. Gu, F. Stepnick, D. M. Poirier, M. B. Tost, P. J. Benning, Y. Chen, T. R. Ohno, José L. Martins, J. H. Weaver, J. Fure e R. E. Smalley, Phys. Rev. B 45, 6348 (1992).

[6] A. F. Hebard, Nature 350, 600 (1991).

[7] S. H. Yang, Chem. Phys. Lett. B 139, 233 (1987).

[8] J. L. Martins e N. Troullier, Phys. Rev. B 46, 1776 (1992).

[9] D. L. Novikov, V. A. Gubanov e A. J. Freeman, Physica C 191, 399 (1992).

[10] H. S. Davidov, Quantum Mechanics, Pergamum Press, Oxford, 559 (1976).

[11] N. W. Ashcroft e N. David Mermin, Solid State Physics, W. B. Saunders Co., Orlando, (1976).

[12] J. Hubbard, Proc. R. Soc. A 276, 238 (1963).

[13] M. C. Gutzwiller, Phys. Rev. Lett. 10, 159 (1963); Phys. Rev. 134, A923 (1964); Phys. Rev. 137, A1726 (1965).

[14] E. A. Guggenheim, Mixtures, p. 38, Oxford University Press, New York, 1952.

[15] R. P. Messner e G. D. Watkins, Phys. Rev. Lett. 25, 656 (1970).

[16] K. Coutinho, S. Canuto, R. Mota e A. Fazzio, Mod. Phys. Lett. B 9, 95 (1995). 
[17] C. C. Chen, S. P. Kelty e C. M. Lieber, Science 253, 886 (1991).

[18] J. P. Lu, Phys. Rev. B 49, 5687 (1994).

[19] J. C. Cechin, R. Mota, S. Canuto e A. Fazzio, "Superconductivity and the Electronic Structure of $A_{x} C_{60}$ ", Workshop on Highly Correlated Systems, LNLS (UNICAMP), dezembro (1994); Thirthy-fifth Sanibel Symposium, University of Florida, USA, março (1995); "Transição Metal-Isolante em Fullerenos Dopados com Metais Alcalinos", XVIII Encontro Nacional de Física da Matéria Condensada, Caxambu/MG, junho (1995).

[20] S. L. Garavelli e A. Ferraz, Mod. Phys. Lett. B 8, 1223 (1994). 